\title{
Effect of Colchicine on Induction of Ploidy and Other Morphological Features of Different Crops: A Review
}

\section{Mushtaq $\mathrm{I}^{1}$, Asghar $\mathrm{R}^{2}$ and Zulfiqar $\mathrm{T}^{3 *}$}

${ }^{1}$ Institute of Botany, University of the Punjab, Pakistan

${ }^{2}$ Department of botany, University of Agriculture Faisalabad, Pakistan

${ }^{3}$ University of Agriculture Faisalabad, Pakistan

*Corresponding author: Taha Zulfiqar, University of Agriculture Faisalabad, Muhallah

\section{Review Article \\ Volume 5 Issue 1}

Received Date: December 28, 2021

Published Date: January 12, 2022

DOI: $10.23880 /$ aabsc- 16000172 saddique pura Narowal, Punjab, Pakistan, Tel: 03367005925; Email: taha.zulfiqar.tz@gmail.com

\section{Abstract}

Colchicine is a mitotic inhibitor and it has been used for induction of tetraploids in different crops. Different concentration of colchicine has been used for different time period in vivo and in vitro. Colchicine is used for such purposes because rate of spontaneously doubling in most species is rare in tissue culture. Treatment of colchicine in in vitro culture plant tissues has been efficiently used to artificially produce polyploids in different plants. In different crops such as vegetables, fruits and flowers tetraploids produce variants with favourable horticultural characteristics like high yield, large fruit size and resistance to different environmental stresses. Tetraploids was induced in vivo by applying colchicine in an aqueous solution, lanolin paste, on apical shoot meristem of seedling and soaked seeds. Colchicine use has been found a very prosperous methodology for genome doubling in a lot of species.

Keywords: Colchicine; Polyploidy plant; Allopolyploids; Tumours

\section{Introduction}

Polyploidy plant production is frequently used in conventional plant breeding, producing polyploids fruits and vegetable used as human food for years. The most important feature of polyploidy is multiplication of genome. Allopolyploids have more than one genome whereas autopolyploid have duplication of a single genome. Different chemicals has been used of the induction of polyploidy in different plants, Colchicine is one of them [1-23]. Colchicine is an important alkaloid which along with its derivatives has many uses e.g. gene expression, gene amplification, anticancer activity and good effect on tumours. Along with such important features, it may also have adverse effect on seed germination and survival of the plant.

Following are some Tables 1-4 of different crops showing effect of colchicine on tetraploids induction:

\begin{tabular}{|c|c|c|c|c|}
\hline SR. No. & Crop & $\begin{array}{c}\text { Colchicine concentration/ } \\
\text { Exposure time/Method }\end{array}$ & Response & Reference \\
\hline 1 & $\begin{array}{c}\text { Orange (Citrus } \\
\text { sinensis) }\end{array}$ & $\begin{array}{c}0.05 \% \& 0.1 \%, 12 \& 24 \text { hours, } \\
\text { In vitro }\end{array}$ & Double number of chromosomes & Wu \& Mooney [23] \\
\hline
\end{tabular}




\begin{tabular}{|c|c|c|c|c|}
\hline 2 & $\begin{array}{l}\text { Pummelo (Citrus } \\
\text { maxima) }\end{array}$ & $\begin{array}{c}0.1 \% \text { \& } 0.05 \%, 4,8,16 \& 32 \\
\text { hours, Indirect organogenesis }\end{array}$ & $\begin{array}{l}\text { Low survival rate, canker } \\
\text { resistant plant, double number of } \\
\text { chromosomes }\end{array}$ & Jude, et al. 2014 \\
\hline \multirow[t]{3}{*}{3} & $\begin{array}{c}\text { Watermelon } \\
\text { (Citrullus lanatus) }\end{array}$ & $\begin{array}{c}0.1 \% \text { and } 0.2 \%, 6,12,18,24 \\
\text { hours, In Vivo }\end{array}$ & $\begin{array}{c}\text { Polyploidy induction, Low } \\
\text { germination and High mortality } \\
\text { rate }\end{array}$ & $\begin{array}{c}\text { Lower and Johnson } \\
\text { [17] }\end{array}$ \\
\hline & & $\begin{array}{c}0.2 \%, 0.4 \% \text { and } 0.6 \%, 3 \text { days, } \\
\text { In vitro }\end{array}$ & $\begin{array}{l}\text { High chlorophyll content, change } \\
\text { in morphology of fruit and plant, } \\
\text { high mortality, flow Cytometry } \\
\text { show high chromosome number }\end{array}$ & Jaskani, et al. [11] \\
\hline & & $0.2,0.4,0.6 \%, 3$ days, In vitro & $\begin{array}{l}\text { Colchicine induced tetraploids } \\
\text { have } 10-12 \text { chloroplast per } \\
\text { guard cell and diploids have } 5-7 . \\
\text { Tetraploids have dark and thick } \\
\text { leaves. }\end{array}$ & Jaskani et al. [12] \\
\hline 4 & $\begin{array}{l}\text { Pear (Pyrus } \\
\text { communis) }\end{array}$ & $\begin{array}{c}0.01 \% \text { and } 0.1 \%, 1,2,4 \text { and } 8, \\
\text { In vitro }\end{array}$ & $\begin{array}{c}\text { Tetraploids and Mixoploids } \\
\text { induction, large stomatal size }\end{array}$ & Kadota, et al. [16] \\
\hline 5 & $\begin{array}{l}\text { Grapevine (Vitis } \\
\text { vinifera) }\end{array}$ & $\begin{array}{c}0,0.1,0.2,0.4 \%, 24 \text { hours, In } \\
\text { vitro }\end{array}$ & $\begin{array}{l}\text { Colchicine has adverse effect on } \\
\text { germination of somatic embryo } \\
\text { and low survival rate. Tetraploids } \\
\text { and triploids was examined in } 0.2 \\
\text { and } 0.4 \% \text { colchicine solution. }\end{array}$ & Acanda, et al. [13] \\
\hline 6 & $\begin{array}{l}\text { Tangerine citrus } \\
\text { (Citrus reticulate) }\end{array}$ & $\begin{array}{c}0.2,0.4,0.8 \%, 12,24 \text { hours, } \\
\text { In vitro }\end{array}$ & $\begin{array}{l}\text { Colchicine have negative effect } \\
\text { on germination of treated seeds. } \\
\text { Stomatal size was highest in } \\
\text { colchicine treated seeds. }\end{array}$ & Surson, et al. [22] \\
\hline
\end{tabular}

Table 1: Colchicine induced mutations in fruit crops.

\begin{tabular}{|c|c|c|c|c|}
\hline $\begin{array}{l}\text { SR. } \\
\text { No. }\end{array}$ & Crop & $\begin{array}{l}\text { Colchicine concentration/ } \\
\text { Exposure time/Method }\end{array}$ & Response & Reference \\
\hline 1 & $\begin{array}{l}\text { Radish } \\
\text { (Raphanus } \\
\text { sativus) }\end{array}$ & $\begin{array}{l}30,60,120 \mathrm{mg} / \mathrm{l}, 5,10,15,20 \\
\quad \& 25 \text { days, In vitro }\end{array}$ & $\begin{array}{l}\text { Reduced regeneration ability of buds and } \\
\text { explant; tetraploids induction; low germination } \\
\text { and low survival; low yield of tetraploids }\end{array}$ & $\begin{array}{c}\text { Manawadu, et al. } \\
{[18]}\end{array}$ \\
\hline 2 & $\begin{array}{c}\text { Tomato } \\
\text { (Solanum } \\
\text { lycopersicum) }\end{array}$ & 8mm, 96 Hours, In vitro & $\begin{array}{l}\text { High mortality in seedlings with low induction; } \\
\text { Low concentration with longer exposure time } \\
\text { was recommended }\end{array}$ & Praca, et al. [20] \\
\hline 3 & $\begin{array}{l}\text { Onion (Allium } \\
\text { cepa) }\end{array}$ & $50 \mu \mathrm{M}, 24$ and 72 hours, & $\begin{array}{l}\text { Colchicine have least chromosome doubling } \\
\text { activity as compared to other mitotic inhibiter } \\
\text { (trifluralin, oryzalin and amprophos-methyl) }\end{array}$ & $\begin{array}{l}\text { Grzebelus and } \\
\text { Adela [15] }\end{array}$ \\
\hline 4 & $\begin{array}{l}\text { Spinach } \\
\text { (Spinacia } \\
\text { oleracea) }\end{array}$ & $\begin{array}{l}1,5,10 \mathrm{mg} / \mathrm{L}, 16,24,32 \\
\text { hours, In vivo }\end{array}$ & $\begin{array}{c}\text { Colchicine have adverse effect on germination } \\
\text { and survival. Chromosome doubling was } \\
\text { identified by Flow Cytometry }\end{array}$ & $\begin{array}{l}\text { Roughani, et al. } \\
\text { [19] }\end{array}$ \\
\hline 5 & $\begin{array}{l}\text { Ginger } \\
\text { (Zingiber } \\
\text { officinale) }\end{array}$ & $\begin{array}{l}0.5,2 \%, 2 \text { hours, Applied on } \\
\text { shoot tips }\end{array}$ & $\begin{array}{l}\text { Tetraploids have large rhizome size, knob } \\
\text { weight, thick shoot and high fiber content }\end{array}$ & Smith, et al. [21] \\
\hline 6 & $\begin{array}{l}\text { Wild potato } \\
\text { (Solanum } \\
\text { commersonii) }\end{array}$ & $\begin{array}{c}3.5,5.0 \text { and } 6.5 \mathrm{mM}, 72 \text { hours, } \\
\text { In vivo }\end{array}$ & $\begin{array}{c}\text { Colchicine have negative effect on germination } \\
\text { and survival. Increase in chromosomal number } \\
\text { was explained by Gigas effect }\end{array}$ & $\begin{array}{c}\text { Gracielle, et al. } \\
{[14]}\end{array}$ \\
\hline
\end{tabular}




\begin{tabular}{|c|c|c|c|c|}
\hline 7 & $\begin{array}{c}\text { Cucumber } \\
\text { (Cucumis } \\
\text { sativus) }\end{array}$ & $\begin{array}{c}250,500,550,1500 \mathrm{mg} / \mathrm{L}, 18, \\
\text { 38hours, In vitro }\end{array}$ & $\begin{array}{l}\text { Higher conc. Of colchicine have adverse effect } \\
\text { on viability of explant Trifluralin and oryzalin } \\
\text { were more effective in chromosome doubling } \\
\text { rather than colchicine }\end{array}$ & Hamed, et al. \\
\hline & & $0.1 \%, 24,48$ hours, In vitro & $\begin{array}{c}\text { Tetraploids have broader leaf area twice than } \\
\text { haploids. Haploids have elongated leaves while } \\
\text { tetraploids have dentate leaves There was clear } \\
\text { difference in flower morphology of tetraploids } \\
\text { and haploids }\end{array}$ & Vesselina, et al. \\
\hline 8 & $\begin{array}{l}\text { Chinese kale } \\
\text { (Brassica } \\
\text { oleracea- } \\
\text { Aboglabra } \\
\text { group) }\end{array}$ & $\begin{array}{c}0.1,0.5,0.251 \text { and } 2 \%, E x \\
\text { vitro }\end{array}$ & Large stomatal size, broader leaf & $\begin{array}{c}\text { Teerarak, et al. } \\
{[10]}\end{array}$ \\
\hline
\end{tabular}

Table 2: Colchicine induced mutations in vegetable crops.

\begin{tabular}{|c|c|c|c|c|}
\hline SR. No. & Crop & $\begin{array}{c}\text { Colchicine concentration/ Exposure } \\
\text { time/Method }\end{array}$ & Response & Reference \\
\hline 1 & $\begin{array}{l}\text { Marigold (Tag- } \\
\text { etes erecta) }\end{array}$ & $0.001 \%, 0.01 \% \& 0.05 \%, 12$ hours & $\begin{array}{l}\text { Maximum shoot number with re- } \\
\text { duced length; slow growth rate; } \\
\text { low survival }\end{array}$ & $\begin{array}{l}\text { Sajjad et al., } \\
2013\end{array}$ \\
\hline 2 & $\begin{array}{l}\text { Ocimum (ocimum } \\
\text { basilicum) }\end{array}$ & $\begin{array}{c}0.05 \%, 0.1 \%, 0.2 \%, 0.5 \% \text { and } 0.75 \%, 6,12 \text {, } \\
24 \text { and } 36 \text { hours, Seed treatment }\end{array}$ & $\begin{array}{c}\text { Large and dark leaf, large size } \\
\text { pollen grains, dark color leaves, } \\
\text { large stomatal length and width } \\
\text { and double number of chloro- } \\
\text { plast in tetraploids as compared } \\
\text { to diploid }\end{array}$ & $\begin{array}{l}\text { Omidbaigi et } \\
\text { al. (2010) }\end{array}$ \\
\hline 3 & $\begin{array}{l}\text { Huang qi (As- } \\
\text { tragalus mem- } \\
\text { branaceus) }\end{array}$ & $0.2 \%, 36$ hours, In vitro & $\begin{array}{l}\text { Large leaf size, large stomatal } \\
\text { size, mixoploids induction }\end{array}$ & $\begin{array}{l}\text { Chen et al. } \\
\text { (2006) }\end{array}$ \\
\hline 4 & Grass & $0.01 \%, 28$ hours, In vitro & $\begin{array}{l}\text { Doubling of chromosomes, } \\
\text { change cytological traits }\end{array}$ & $\begin{array}{l}\text { Pinheiro et } \\
\text { al. }(2000)\end{array}$ \\
\hline
\end{tabular}

Table 3: Colchicine induced mutations in flower crops.

\begin{tabular}{|c|c|c|c|c|}
\hline $\begin{array}{c}\text { SR. } \\
\text { No. }\end{array}$ & Crop & $\begin{array}{c}\text { Colchicine } \\
\text { concentration/ } \\
\text { Exposure time/ } \\
\text { Method }\end{array}$ & Response & Reference \\
\hline 1 & $\begin{array}{c}\text { Ashwagandha } \\
\text { (Withania } \\
\text { somnifera) }\end{array}$ & $\begin{array}{c}0.5 \%, 3,6,8 \text { hours, In } \\
\text { vitro }\end{array}$ & $\begin{array}{c}\text { Stomatal density decreases with in } \\
\text { stomatal size in colchicine induced } \\
\text { tetraploids. }\end{array}$ & Vidya, et al. [5] \\
\hline 2 & $\begin{array}{c}\text { Indian sarsaparilla } \\
\text { (Hemidesmus } \\
\text { indica) }\end{array}$ & $\begin{array}{c}0.5,1,2 \text { and } 5 \mathrm{mg} / \mathrm{L}, 16 \\
\text { and } 8 \text { hours, In vitro }\end{array}$ & $\begin{array}{c}\text { Colchicine have more effect on shoots } \\
\text { as compared to roots nodal explants. } \\
\text { in solid medium as compared to liquid } \\
\text { medium but tetraploids induction was } \\
\text { high in liquid medium. }\end{array}$ & Nagahatenna, et al. [3] \\
\hline
\end{tabular}




\begin{tabular}{|c|c|c|c|c|}
\hline 3 & $\begin{array}{c}\text { Jujuba (Zizyphus } \\
\text { jujuba) }\end{array}$ & $\begin{array}{c}0.01 \% 0.03 \% 0.1 \% \\
\text { and } 0.3 \%, 24,48,72 \\
\text { and } 96 \text { hours, In vitro }\end{array}$ & $\begin{array}{c}\text { Large stomatal size, double number of } \\
\text { chloroplast in guard cells, change in } \\
\text { morphology, doubling of chromosome } \\
\text { number }\end{array}$ & Gu, et al. [4] \\
\hline 4 & $\begin{array}{c}\text { Mopane } \\
\text { (Colophospermum } \\
\text { mopane) }\end{array}$ & $\begin{array}{c}0.05 \%, 0.1 \% \text { and } 1 \%, \\
\text { Soaking seeds }\end{array}$ & $\begin{array}{c}\text { Chimeras formation, low survival } \\
\text { percentage, high mortality, slow growth } \\
\text { rate, large lateral branches with smaller } \\
\text { leaflets }\end{array}$ & Rubuluza, et al. [1] \\
\hline 5 & $\begin{array}{c}\text { Oil palm (Elaeis } \\
\text { guineensis) }\end{array}$ & $\begin{array}{c}2.5 \mathrm{mM} \text { to } 10.0 \mathrm{mM}, 6 \\
\text { to } 48 \text { hours, In vitro }\end{array}$ & $\begin{array}{c}\text { Tetraploids and Mixoploids induction, } \\
\text { change in genome, change in stomatal } \\
\text { index }\end{array}$ & Madon, et al. [2] \\
\hline
\end{tabular}

Table 4: Colchicine induced mutations in medicinal plants.

\section{Conclusion}

Application of colchicine on different crops reported mutation in different crops in the form of chromosomal doubling. Most effective method is in vitro and with low concentration of colchicine. High concentration of colchicine with longer exposure time may adversely affect the survival rate of crop. Morphological changes such larger leaf size, larger flower size more chloroplast per guard cell also confirmed the induction of polyploidy.

\section{References}

1. Rubuluza T, Nikolova RV, Smith MT, Hannweg K (2007) In vitro induction of tetraploids in Colophospermum mopane by colchicine. South African J Bot 73: 259-261.

2. Madon M, Clyde MM, Hashim H, Yusuf MY, Mat H (2005) Polyploidy induction of oil palm through colchicine and oryzalin treatments. J Oil Palm Res 17: 110-123.

3. Nagahatenna DSK, Peiris SE (2008) Modification of plant architecture of Hemidesmusindica (L.) R. Br. (Iramusu) by in vitro colchicine treatment. Trop Agri Res 20: 234242.

4. Gu XF, Yang AF, Meng $\mathrm{H}$, Zhang JR (2005) In vitro induction of tetraploids plants from diploidc Zizyphus jujube Mill. cv. Zhanhua. Plant Cell Rep 24(11): 671-676.

5. Vidya KM, Saraswathi G, Kumar BMV (2013) Induction and identification of autotetraploidy in ashwaghandha (Withania somnifera). Asia J Horti 8(1): 32-35.

6. Sajjad Y, Jaskani MJ, Mehmood A, Ahmad I, Abbas H (2013) Effect of colchicine on in vitro polyploidy induction in African Marigold. Pak J Bot 45(3): 1255-1258.

7. Pinheiro AA, Pozzobon MT, do Valle CB, Penteado MIO, Carneiro VTC (2000) Duplication of the chromosome number of diploid Brachiaria brizantha plant using colchicine. Plant Cell Rep 19(3): 274-278.

8. Omidbaigi R, Mirzaee M, Hassani ME, Moghadam MS (2010) Induction and identification of polyploidy in basil (OcimumbasilicumL.) medicinal plant by colchicine treatment. Int J Plant Prod 4(2): 87-98.

9. Chen LL, Gao SL (2006) In vitro tetraploids induction and regeneration of tetraploids from mixoploids Astragalus membranaceus. Sci Hortic 112: 339-344.

10. Teerarak M, Thitavasanta S, Khamchutr S (2006) Preliminary studies on the creation of anatomical changes by colchicine treatment in Chinese kale (Brassica alboglabra). KMITL Sci J 6(2): 2.

11. Jaskani MJ, Kwon SW, Kin DH (2005) Flow Cytometry of DNA content of colchicine treated watermelon as a screening method at M1 stage. Pak J Bot 37(3): 685-695.

12. Jaskani MJ, Kwon SW, Hussain Z, Khan IA (2007) Breeding polyploidy watermelon: Induction, identification and seed germination of tetraploids. Int Symp Prosp Horti Industri Pak.

13. AcandaY, Martinez O,Gonzalez MV,Prado MJ, Rey M (2015) Highly efficient in vitro tetraploid plant production via colchicine treatment using embryogenesis suspension culture in grapevine (Vitis vinefera). Plant Cell Tiss Org Cult 123(3): 547-555.

14. Gracielle L, Tome O, Silva AB, Augusto C, Pinto BP, et al. (2016) Colchicine and oryzalin effect on tetraploids induction and leaf anatomy of Solanum commersonii sp. Cienc Rurl 46(11): 1973-1979.

15. Grzebelus Ewa, Adamus A (2004) Effect of anti-mitotic agents on development and genome doubling of gynogenic onions (Allium cepa L.) embryos. Plant sci 167(3): 569-574.

16. Kadota M, Niimi Y (2002) In vitro induction of 
tetraploid plants from a diploid Japanese pear cultivar (Pyruspyrifolia N. cv. Hosui). Plant Cell Rep 21(3): 282286.

17. Lower RL, Johnson KW (1969) Observations on sterility of induced autotetraploid watermelons. J Amer Soc Hort Sci 94: 367-369.

18. Manawadu IP, Dahanayake N, Senanayake S (2016) Colchicineinducedtetraploidsofradish(RaphanusSativus L.). Trop Agri Res Ext 19: 2016.

19. Roughani A, Miri SM, Kashi AK, Khiabani BN (2017) Increase the ploidy level in spinach (Spinacia oleracea L.) using mitotic inhibitor. J Plant Cell Bitech Mol Bio 18(3-4): 124-130.

20. Praca MM, Carvalho CR, Clarindo WR (2009) A practical and reliable procedure for in vitro induction of tetraploids tomato. Sci Horti 122: 501-505.

21. Smith MK, Hamill SD, Gogel BJ, Severn AA (2004) Ginger (Zingiber officinale) auoetraploid with improved processing quality produced by an in vitro colchicine treatment. Australian J Exp Agr 44(10): 1065-1072.

22. Surson S, Sitthaphanit S, Wongma N (2015) In vivo induction of tetrapoid in Tangerine citrus plants (Citrus reticulate Blanco) with the use of colchicine. Pak J Biol Sci 18(1): 37-41.

23. Wu JH, Mooney P (2002) Autotetraploid tangor plant regeneration from in vitro citrus somatic embryogenic callus treated with colchicine. Pl Cell Tiss Org Cult 70: 99-104. 